REVUE CRITIQUE

\title{
LA TRAME DE LA LOGIQUE FLOUE ET L'USURE DU TEMPS*
}

\author{
Caroline EHRHARDT
}

Les sciences déductives étaient jusqu'à présent restées à l'écart du renouveau apporté par les approches sociologiques à l'étude des sciences contemporaines ${ }^{1}$. L'ouvrage de Claude Rosental les sort de leur isolement en s'attachant à mettre en évidence, par une enquête sociologique, le processus d'accréditation d'un théorème de logique mathématique : le théorème d'Elkan. L'ouvrage ne se réduit cependant pas à une étude empirique : la nouveauté de l'objet impose à l'auteur de développer un cadre méthodologique et des outils inédits pour le décrire. La Trame de l'évidence s'avère ainsi être un ouvrage magistral fort réussi, qui ouvre de nouvelles perspectives quant à l'analyse des pratiques mathématiques.

Le livre s'ouvre sur une étude historiographique où sont répertoriés et analysés quelques travaux d'inspiration sociologique consacrés aux sciences déductives. Selon Rosental, à une littérature philosophique considérable qui traite d'une logique idéalisée, conçue comme science du raisonnement, la sociologie des sciences s'est contentée d'opposer une discussion de principe portant sur l'existence de plusieurs logiques discordantes rattachées à des facteurs historiques et culturels distincts ${ }^{2}$. La logique considérée comme une activité mathématique particulière a fait l'objet d'études de textes qui cherchent à faire apparaître le raisonnement de leur auteur en le considérant comme une pratique conceptuelle ${ }^{3}$; le travail logique et celui des logiciens demeurent le plus souvent idéalisés par les sociologues, puisqu'ils ne sont envisagés qu'à titre de processus immatériels et solitaires ${ }^{4}$. Des pistes de recherche sont cependant fournies par l'étude des pratiques concrètes liées au travail d'abstraction ${ }^{5}$ ou encore, par celle du rôle joué par l'enseignement dans

* À propos de : Claude Rosental, La Trame de l'évidence. Sociologie de la démonstration en logique, Paris, Presses universitaires de France, 2003, $15 \times 22,367$ p., index (Sciences, modernités, philosophies).

1. À côté de l'imposante littérature consacrée aux sciences expérimentales, les analyses concernant les mathématiques et la logique restent marginales. On peut néanmoins citer les travaux de MACKeNZIE, 1993.

2. RosentAL, 2003, p. 42-51, à propos de BLOOR, 1982 ; RoSENTAL, 2003, p. 67, à propos de NYE, 1990. Notons cependant ici que ces ouvrages traitent de la logique en la considérant comme une science du raisonnement et non comme discipline mathématique.

3. Rosental, 2003, p. 51-53, à propos de Pickering et StePHANIDES, 1992.

4. Rosental, 2003, p. 68-71, à propos de LiVINGSTON, 1985.

5. Rosental, 2003, p. 58-61, à propos de Brian, 1994. 
la constitution de savoirs et savoir-faire tacites en mathématique 6 . De plus, les études réalisées par des mathématiciens professionnels soulignent l'existence d'arguments extra-mathématiques dans les processus d'évaluation des démonstrations ${ }^{7}$.

La première partie de l'ouvrage de Rosental s'achève sur la description du forum électronique dans lequel se sont déroulés les débats autour du théorème d'Elkan et sur le compte rendu d'une enquête réalisée en 1992 sur l'enseignement de la logique. Ces deux études préliminaires visent à familiariser le lecteur avec le vocabulaire logique et le fonctionnement du forum ; l'auteur s'attache également à mettre en évidence les aspects les plus concrets du débat sur Internet (saisie manuelle des textes, processus d' archivage et de filtrage... ${ }^{8}$ ) et à dégager les pratiques propres à cette forme d'apprentissage de la logique ${ }^{9}$.

L'enquête de Claude Rosental porte sur un résultat publié en 1993 par l'universitaire californien Charles Elkan à l'occasion d'un colloque sur l'intelligence artificielle ; l'article de ce chercheur, intitulé « The paradoxal success of fuzzy $\operatorname{logic}{ }^{10}$ », visait à remettre en cause une théorie logique appelée « logique floue », en démontrant que celle-ci se réduisait en fait à la logique binaire classique. Rosental s'attache à étudier la controverse provoquée par ce résultat sur une durée d'environ un an, ainsi que les différentes phases de l'élaboration de l'article d'Elkan. La deuxième partie de La Trame de l'évidence est entièrement consacrée à la controverse qui s'est déroulée sur le forum électronique « comp.ai.fuzzy » à propos de l'article d'Elkan entre août 1993 et août 1994. Rosental examine tout d'abord les messages relatifs à la validité et à la valeur de la preuve (chap. III) qui mettent en jeu des démonstrations formelles, puis porte son attention sur les autres types d'arguments qui interviennent dans le débat (chap. IV).

Le premier constat dressé par l'auteur est celui d'un « corpus cacophonique », où les intervenants partagent un mode de questionnement et un vocabulaire communs (ce qui conforte l'hypothèse formulée précédemment d'une certaine homogénéité des savoirs tacites), sans pour autant s'accorder sur la validité de la démonstration d'Elkan ni sur la nature de l'erreur qu'il a commise. La logique floue ne fait l'objet d'aucune définition consensuelle, et les acteurs mobilisent des axiomatiques concurrentes, prenant en compte ou non certains principes fondamentaux (comme celui du tiers exclu ${ }^{11}$ ). L'attention de l'auteur se porte ensuite sur les modalités matérielles des interventions; il s'agit d'actes d'écriture concrets visant à produire de nouveaux textes démonstratifs et à reformuler la preuve d'Elkan en «faisant apparaître » certains principes logiques entre les lignes : les auteurs essaient de convaincre de l'utilisation implicite du principe du tiers exclu ou encore de celui de distributivité en les énonçant au sein même de la démonstration

6. Rosental, 2003, p. 53-56, à propos de WARWICK, 1992 et 1993.

7. Rosental, 2003, p. 61-66, à propos de Davis et Hersh, 1981.

8. Rosental, 2003, p. 81-86.

9. Rosental, 2003, p. 86-105.

10. ELKAN, 1993.

11. RosenTAL, 2003, p. 109-133. 
et en explicitant leur utilisation dans son déroulement. De telles pratiques ne constituant pas selon Rosental des démonstrations au sens mathématique du terme, il les définit comme des pratiques de « dé-monstrations » pour souligner le caractère visuel des argumentations produites. Il montre ensuite qu'elles ont pour effet des ajustements constants du statut de la preuve d'Elkan et de celui de ses substituts ${ }^{12}$. La dimension visuelle de la controverse sur le forum se manifeste également par des stratégies de visibilité des messages : certaines traductions de la démonstration originelle, reproduites dans d'autres messages ou accessibles grâce à des liens hypertexte, offrent un accès privilégié aux intervenants qui n'ont pas eu le temps ou la possibilité de se procurer l'article d'Elkan. Du fait de contraintes temporelles et matérielles éprouvées par les protagonistes, les points de vue sur l'article initial sont donc structurés par les transpositions induites par de tels messages ${ }^{13}$.

Les débats sur l'article d'Elkan ne se limitent cependant pas au domaine de la logique «pure » : certaines interventions portent sur les applications de la logique floue, d'autres sur l'hostilité des chercheurs en intelligence artificielle à cette logique, d'autres enfin mettent l'accent sur les particularités culturelles de cette théorie, qu'ils rattachent à un mode de pensée qualifié d'oriental. En analysant ces messages et les réponses qu'Elkan leur adresse, Rosental met ici en évidence un grand nombre de registres «dé-monstratifs » combinés dans les textes des intervenants, dont il explique la variété par la multiplicité des points de vue concernant la logique floue et des domaines technologiques dans lesquels elle intervient ${ }^{14}$.

Après l'étude locale et à court terme de la controverse sur le forum Internet, Rosental élargit son champ d'investigation dans la troisième partie de l'ouvrage : il s'agit désormais de situer les messages du forum par rapport à des dynamiques à plus long terme concernant l'ensemble des acteurs de la logique floue. Le chapitre $\mathrm{V}$ a ainsi pour objet de retracer l'historique de la publication d'Elkan ; à cet effet, l'auteur a recours à des entretiens avec des chercheurs en logique floue et à des observations lors d'un colloque sur cette théorie. Il met ainsi en lumière l'importance du «travail d'accompagnement» auquel se livrent les acteurs afin de défendre leurs "dé-monstrations », en reformulant leurs thèses ou en cherchant à mettre en avant leur point de vue ${ }^{15}$. Plus encore, la préparation d'un article n'apparaît pas comme une activité solitaire, mais au contraire comme soumise à de nombreuses interactions : les représentations qu'a Elkan de la logique floue sont en fait confortées par le grand nombre d'acteurs qui partagent son point de vue ${ }^{16}$. Enfin, le processus de sélection des articles lors des colloques ne répond pas à des critères absolus de validité des preuves : aux dires des acteurs eux-mêmes, il est conditionné par le savoir-faire des rapporteurs, l'instauration de quotas ou encore les objectifs du colloque ${ }^{17}$.

12. Rosental, 2003, p. 133-149.

13. Rosental, 2003, p. 149-156.

14. Rosental, 2003, p. 157-197.

15. Rosental, 2003, p. 202-215.

16. Rosental, 2003, p. 215-228.

17. Rosental, 2003, p. 228-248. 
Les deux derniers chapitres de l'ouvrage rendent compte de la clôture de la controverse et de l'accréditation du théorème. Le déplacement du débat vers des revues en modifie la dynamique : à la multiplicité des points de vue fait place la riposte structurée d'un groupe de chercheurs considérés comme les porte-parole de la logique floue, et dont l'intervention se situe dans le contexte plus large d'une opposition commune à une certaine définition de cette théorie, antérieure à l'article d'Elkan ${ }^{18}$. Ces chercheurs, habitués à répondre aux critiques à propos de leur discipline, n'ont pas eu à improviser de réponses nouvelles et propres aux discussions sur le théorème d'Elkan, alors que celui-ci avait tendance à personnaliser ses interventions afin d'obtenir des points d'accord locaux. De plus, la comparaison des différentes versions de l'article permet à Rosental de mettre en évidence les corrections apportées à la suite des débats, visant à rendre la preuve plus résistante aux critiques. L'auteur met également en lumière une stratégie d'écriture particulière : Elkan joue sur la polysémie des expressions employées pour limiter les possibilités de conflit tout en ralliant à ses thèses des lecteurs ayant des points de vue divergents ${ }^{19}$. Au final, la clôture de la controverse ne s'accompagne pas d'un véritable consensus, ni de la victoire d'un camp sur l'autre : l'accord sur la validité du théorème d'Elkan repose en fait sur les représentations très diverses de la preuve qu'autorise la polysémie du texte. Ainsi, pour Rosental, la validité de ce théorème constitue non pas un énoncé universel mais « un énoncé collectif ${ }^{20}$ ».

Les analyses sociologiques concernant les sciences déductives étant très peu nombreuses, l'un des premiers mérites du livre est de fournir une telle enquête sur une activité scientifique, la logique, réputée immatérielle et strictement individuelle, donc inobservable par le sociologue. À cet effet, Rosental s'attache à mettre en évidence la dimension collective et matérielle de l'activité : de nombreux échanges ont lieu entre les acteurs, que ce soit à l'écrit ou à l'oral, et des dispositifs matériels (ordinateurs, tableaux...) entrent en jeu. L'étude accorde également une importance particulière aux pratiques concrètes auxquelles se livrent les acteurs, y compris dans les détails précis des démonstrations, tout en s'interdisant de donner une définition restrictive de ce qu'est « la » logique. À la suite de ses observations, Rosental met ainsi en lumière une image inhabituelle de cette science, plus proche de la description que font les mathématiciens professionnels de leur travail quotidien que de celle des enquêtes normatives : il s'agit d'une activité démonstrative concrète réalisée essentiellement par écrit. La pratique logique n'est pas envisagée ici comme une succession de raisonnements abstraits que l'on couche ensuite sur le papier : à la suite de Jack Goody (et d'Éric Brian), Claude Rosental affirme que l'acte d'écriture participe pleinement à la production des objets logiques ${ }^{21}$.

18. ROSENTAL, 2003, p. 249-264.

19. Rosental, 2003, p. 280-316.

20. Rosental, 2003, p. 316-331. Pour la notion d'énoncé collectif, l'auteur se réfère ici à BOUREAU, 1989. On peut également consulter GoldSTEIN, 1989.

21. Rosental, 2003, p. 145. 
En considérant la logique comme un objet sociologique à part entière, Rosental parvient également à démontrer que la validité d'une preuve logique ne découle pas seulement de sa présentation formelle; de même, cette validité n'est pas non plus garante de la reconnaissance de la démonstration par la communauté des chercheurs $^{22}$. Enfin, la logique elle-même n'est pas considérée ici comme un dispositif transparent : elle n'est pas donnée d'avance parmi les acteurs et une partie de leur activité réside dans la négociation de sa définition et de ses propriétés. Notons ici que, si l'approche envisagée est différente, les conclusions de l'auteur sur la mise en suspens de l'universalité des formes de démonstrations et sur la matérialité des sciences déductives rejoignent en partie celles de certains travaux récents en histoire des mathématiques ${ }^{23}$. À l'inverse de ces approches, le processus d'accréditation du théorème analysé par Rosental semble toutefois laisser de côté les propriétés de la logique qui ne relèveraient pas de la pratique, comme par exemple sa vocation à l'universalité ${ }^{24}$. Par contre, les conclusions de l'auteur atténuent largement les différences supposées et presque toujours ininterrogées dans l'étude des sciences entre sciences déductives et sciences expérimentales et lui permettent, après avoir précisé ce en quoi consistent les pratiques logiques, de s'inspirer de la méthodologie de la sociologie des sciences.

La Trame de l'évidence apparaît en effet davantage comme un ouvrage de sociologie ou d'ethnologie des pratiques scientifiques que comme une étude portant sur la logique considérée comme une science déductive, la spécificité de l'objet n'intervenant ici que dans la mesure où il s'agit de rendre compte de pratiques théoriques d'abstraction et non de pratiques expérimentales. Cet ouvrage rejoint les principes directeurs de la sociologie des pratiques scientifiques à plusieurs niveaux. D'une part, en s'intéressant au processus d'accréditation d'un théorème, Rosental étudie un phénomène scientifique encore incertain, c'est-à-dire « en train de se faire », pour reprendre une expression ambiguë commune à l'épistémologie et à la sociologie, que l'auteur considère ici à la manière de Bruno Latour ${ }^{25}$. D'autre part, son analyse vise à saisir la diversité des acteurs et des démarches qui concourent à l'accréditation du théorème : l'auteur ne raisonne pas en termes de groupes de chercheurs, mais accorde une place importante à l'identité des acteurs, en insistant sur les positions inégales qu'ils occupent, sur la multiplicité des interprétations et sur la personnalisation de certains échanges. Notons cependant que, dans la partie consacrée au forum de discussion sur Internet, tous les intervenants sont également pris au sérieux par le sociologue. En outre, de même que l'ethnométhodologie des sciences ne considère pas la nature et la réalité comme des données préexistantes que le scientifique entend décrire, Rosental se garde de poser par avance une définition de ce que serait la logique ${ }^{26}$ : celle-ci fait au

22. RosentaL, 2003, p. 109-133, 202-215 et 228-248.

23. À ce propos, sur les périodes moderne et contemporaine, voir par exemple les travaux de BRIAN, 1994 et 1995 ; Cifoletti, 1995 et 1998 ; Goldstein, 1989 et 1995.

24. Nous nous appuyons ici sur CIFOLETTI, 1998.

25. LATOUR, 1989.

26. Rosental, 2003, p. 14. 
contraire l'objet de définitions multiples, constamment renégociées par les interventions des acteurs ${ }^{27}$.

À la suite de Bruno Latour et Steve Woolgar ${ }^{28}$, Claude Rosental insiste ainsi sur l'importance des pratiques de lecture et d'écriture ; de même, la mise en évidence de la coexistence de différents types de «dé-monstrations » rappelle les résultats avancés par Karin Knorr-Cetina ${ }^{29}$ à propos des raisonnements des chercheurs : ceux-ci ne reposent pas exclusivement sur des arguments rationnels, et font au contraire intervenir des éléments aussi variés que l'exhibition des dispositifs fonctionnant grâce à la logique floue, la thèse du complot contre cette logique ou l'appel à la confiance du lecteur ${ }^{30}$.

Si l'ouvrage de Claude Rosental présente de nombreux points communs avec l'ethnométhodologie des sciences, il s'en distingue néanmoins par certains aspects importants. Certes, l'analyse de Rosental rompt avec la dichotomie entre facteurs sociaux et cognitifs, comme le fait l'ethnométhodologie des sciences, mais en allant plus loin : la démonstration d'Elkan est envisagée comme un quasi-objet, ce qui autorise l'auteur à saisir l'autonomie relative de l'objet logique considéré et les éléments d'hétéronomie qu'il comporte ainsi ${ }^{31}$. L'auteur parvient en outre à traiter dans un même mouvement les deux aspects, en montrant que des représentations de la logique et des coalitions d'acteurs se stabilisent simultanément au cours du processus. Enfin, on retrouve dans La Trame de l'évidence certains thèmes centraux des recherches ethnométhodologiques antérieures. De plus, les analyses de Rosental ne reposent pas exclusivement sur les observations des acteurs, l'une des sources les plus importantes de l'auteur étant le dépouillement des archives du forum « comp.ai.fuzzy ». Par ailleurs, l'auteur n'affiche pas une ignorance délibérée de la logique floue en tant que science : son enquête est informée solidement en logique et en mathématiques ${ }^{32}$. Il nous semble également important de noter que si l'étude de Rosental s'apparente à une analyse de controverse, elle évite cependant une lecture relativiste sociale de la stabilisation de ce théorème : la validation du théorème repose sur les ajustements successifs qu'il a subis et non sur des facteurs supposés externes.

La Trame de l'évidence réalise le tour de force d'adapter un questionnement jusqu'ici réservé aux sciences expérimentales à la production d'énoncés logiques. L'auteur insiste d'ailleurs à de nombreuses reprises sur les représentations largement répandues de cette discipline en tant qu'activité abstraite et immatérielle, qu'il oppose aux similarités soulignées par son étude entre pratiques expérimentales et théoriques : la démarche adoptée tend à atténuer plutôt qu'à préciser les spécificités de son objet d'étude. Rosental n'affronte cependant pas ici le corps le

27. Rosental, 2003, p. 117-129 et 157-168.

28. LATOUR et WOOLGar, 1988.

29. KNORR-CETINA, 1981.

30. Rosental, 2003, p. 194-195.

31. Rosental, 2003, p. 281-289.

32. Il précise cependant que celle-ci aurait constitué un «handicap » sans la formation reçue parallèlement en sociologie des sciences expérimentales. RosENTAL, 2003, p. 14. 
plus abstrait de la logique formelle, et donc ce qu'on envisage comme le «noyau dur » des sciences déductives : en effet, la logique floue connaît de nombreuses applications informatiques et technologiques, ce qui ancre la discipline dans la « réalité concrète» (il est d'ailleurs aujourd'hui banal d'utiliser un lave-linge programmé selon les principes de la logique floue ${ }^{33}$ ). Sur ce point, La Trame de l'évidence est un ouvrage paradoxal : il entend montrer que la logique ne présente pas les spécificités qu'on lui prête habituellement, mais considère un domaine particulier, plus « concret » et moins « pur », et lui applique une méthodologie qui tend à gommer ses spécificités autres que matérielles.

Mais outre les modalités scripturales de leur mise en œuvre, la logique et les mathématiques se distinguent des autres sciences par leur vocation à durer et leur prétention à être universellement valides ${ }^{34}$. L'étude de Rosental, fondée sur des observations contemporaines, se déploie dans une temporalité courte et fait intervenir un nombre somme toute limité de chercheurs liés à la logique floue. Le critère qui pousse l'auteur à attribuer au théorème le statut de connaissance certifiée, après un an environ de débats, repose sur la stabilisation relative des points de vue des intervenants, fondée sur des représentations divergentes du sens de la preuve et de la notion même de validité. L'accréditation du théorème signifie donc ici que le théorème n'a pas été catégoriquement invalidé et qu'il a accédé à une certaine reconnaissance. Il nous semble cependant un peu excessif d'en faire pour autant une « connaissance certifiée » sans prendre en compte sa postérité à plus long terme : un tel statut réside en effet selon nous dans les utilisations et citations successives qu'en feront éventuellement les logiciens pendant une ou plusieurs générations ${ }^{35}$.

D'autre part, Rosental insiste sur la construction simultanée du résultat d'Elkan et de certaines propriétés de la logique floue à travers les négociations. Les particularités de ce domaine de recherche sont signalées, mais n'apparaissent pas comme déterminantes dans l'étude. Il s'agit pourtant d'une discipline relativement récente, qui ne dispose pas d'un corps de connaissances partagées par tous les chercheurs et dont la crédibilité n'est pas affirmée. Il est donc possible que les définitions et propriétés qu'elle met en jeu soient plus flexibles que celles d'une discipline plus solidement constituée. Dès lors, il conviendrait d'examiner à nouveau frais les enjeux des différentes interprétations du théorème quant à la redéfinition des frontières de la jeune discipline ${ }^{36}$.

Au-delà de l'objet étudié, l'une des nouveautés du livre de Rosental réside dans le choix des sources utilisées : en plus des observations dans les universités et les colloques, et des entretiens avec les chercheurs, l'auteur appuie son enquête sur le dépouillement des archives d'un forum de discussion sur internet, dont il détaille à cette occasion le fonctionnement. Ce forum permet à tout individu disposant d'une

33. Rosental, 2003, p. 157-168.

34. Cifoletti, 1998.

35. À ce propos, concernant la postérité à long terme d'un théorème de théorie des nombres, voir GoldSTEIN, 1995, p. 5-17. Cette question est également abordée dans BRIAN, 1995, p. 93-95.

36. Pour plus de précision sur la logique floue, ainsi qu'une mise au point méthodologique, on peut consulter RosentaL, 1998. 
connexion de lire et d'écrire des messages consacrés à la logique floue ; de ce fait, les débats qui s'y déroulent sont relativement ouverts, puisque les interventions ne font l'objet d'aucune sélection et qu'elles sont moins élaborées que les textes destinés à paraître dans des revues. De plus, la médiation du forum électronique autorise une forme d'échanges particulière : les acteurs intercalent fréquemment les passages des démonstrations antérieures qu'ils contestent entre les lignes de leurs propres textes, et les arguments développés sortent parfois largement du champ de la démonstration logique formelle. Ce forum offre ainsi un lieu d'échanges informels entre les intervenants, qui permet un dialogue personnalisé entre certains d'entre eux et l'utilisation d'une large gamme de ce que Rosental qualifie de « dé-monstrations ».

L'ouvrage de Rosental ne se limite cependant pas ici à la description d'une forme particulière de communication scientifique ; il montre également que l'organisation des échanges induit un mode particulier de production de connaissances. En effet, les représentations de l'article d'Elkan sont en partie forgées par les présentations et reformulations proposées sur le forum, fondées sur des registres démonstratifs divers et prenant appui sur les messages antérieurs : la signification de la preuve d'Elkan, sa validité et son statut en tant que démonstration logique sont ainsi perpétuellement reformulés et négociés au cours des échanges. Par ailleurs, les stratégies de visibilité développées par certains des auteurs permettent de donner plus de poids à leurs messages dans la formation des points de vue sur l'article d'Elkan. Le forum Internet « comp.ai.fuzzy » n'est donc pas seulement un lieu de dialogues : il opère également comme un lieu d'élaboration de savoirs, dont le mode d'organisation spécifique oriente la production.

Ainsi, La Trame de l'évidence constitue une belle application d'une démarche sociologique à l'étude du travail logique, qui montre que les sciences déductives ne sont pas imperméables au questionnement sociologique. L'ouvrage parvient à prendre en compte la dimension pratique de la logique sans pour autant sombrer dans le relativisme social. L'utilisation des archives d'un forum Internet ouvre en outre de nouvelles pistes de recherche, qui permettent de questionner les liens entre production et lieux d'élaboration des connaissances scientifiques. Centré sur le processus d'accréditation d'un théorème, l'ouvrage traite également du passage de la production locale des savoirs à la reconnaissance par les pairs. Il offre ici une réponse originale, fondée sur la notion d'énoncé collectif. Mais l'objet d'étude n'est saisi qu'au moyen de pratiques quasi immédiates et se trouve ainsi limité à une temporalité très courte : il reste à saisir ce que la démonstration, une fois la « dé-monstration » acquise, doit à l'épreuve du temps social. Ce pourrait être l'objet d'une suite que donnerait Rosental à cette première enquête, déjà neuve et plutôt efficace.

Caroline EHRHARDT

Centre Alexandre-Koyré

Pavillon Chevreul

57, rue Cuvier - F-75231 Paris Cedex 05 caroline.ehrhardt@mageos.com

(juin 2003). 


\section{LISTE DES RÉFÉRENCES}

Bloor (David), 1982, Sociologie de la logique. Les limites de l'épistémologie, Paris, Pandore.

Boureau (Alain), 1989, «Propositions pour une histoire restreinte des mentalités », Annales. Économies, sociétés, civilisations, 6, nov.-déc., p. 1491-1504.

BRIAN (Éric), 1994, La Mesure de l'État. Administrateurs et géomètres au XVIII' siècle, Paris, Albin Michel.

BRIAN (É.),1995, « Le livre des sciences est-il écrit dans la langue des historiens ? », in LEPETIT (Bernard), éd., Les Formes de l'expérience. Une autre histoire sociale, Paris, Albin Michel, p. 85-98.

CifoletTi (Giovanna), 1995, «La question de l'algèbre. Mathématique et rhétorique des hommes de droit dans la France du XVI ${ }^{\mathrm{e}}$ siècle », Annales. Histoire, sciences sociales, 6, nov.-déc., p. 1385-1416.

Cifoletti (G.), 1998, «L'histoire culturelle des mathématiques », in Guesnerie (Roger) et HaRtog (François), dir., Des sciences et des techniques. Un débat, Paris, Armand Colin-EHESS, p. 163-170.

Davis (Philip J.) et Hersh (Reuben), 1981, The Mathematical Experience, Boston, Birkhäuser.

ElKan (Charles), 1993, « The paradoxal success of fuzzy logic », in National Conference on Artificial Intelligence, Proceedings of the Eleven National Conference on Artificial Intelligence, sponsored by the American Association for Artificial Intelligence (AAAI), 1993, Menlo Park, CA/Cambridge, MA, The American Association for Artificial Intelligence Press/Massachussets Institute of Technology Press, p. 698-703.

GoLDSTEIN (Catherine), 1989, «Le métier des nombres aux XVIII ${ }^{\mathrm{e}}$ et XIX ${ }^{\mathrm{e}}$ siècles », in SERRES (Michel), éd., Éléments d'histoire des sciences, Paris, Bordas, p. 275-295.

Goldstein (C.), 1995, Un théorème de Fermat et ses lecteurs, Saint-Denis, Presses universitaires de Vincennes.

Knorr-Cetina (Karin), 1981, The Manufacture of knowledge. An essay on constructivist and contextual nature of science, Oxford, Pergamon Press.

Latour (Bruno), 1989, La Science en action, Paris, La Découverte.

Latour (Bruno) et Woolgar (Steve), 1988, La Vie de laboratoire. La production des faits scientifiques, Paris, La Découverte.

LiVInGSTON (Eric), 1985, The Ethnomethodological Foundations of mathematics, Londres, Routledge.

MACKENZIE (Donald), 1993, « Negociating arithmetic, constructing proof. The sociology of mathematics and information technology », Social Studies of Science, vol. XXIII, p. 37-65. 
NYE (Andrea), 1990, Words of power. A feminist reading of the history of logic, Londres, Routledge.

Pickering (Andrew) et STEHANIDES (Adam), 1992, « Constructing quaternions. On the analysis of conceptual practice », in PICKERING (Andrew), éd., Science as practice and culture, Chicago, IL, Chicago University Press, p. 139-167.

Rosental (Claude), 1998, « Histoire de la logique floue. Une approche sociologique des pratiques de démonstration », Revue de synthèse, $4^{\mathrm{e}}$ sér., 4, oct.-déc., p. 575-602.

Rosental (C.), 2003, La Trame de l'évidence. Sociologie de la démonstration en logique, Paris, Presses universitaires de France.

WARwICK (Andrew), 1992, «Cambridge mathematics and Cavendish physics. Cunningham, Campbell and Einstein's relativity theory 1905-1911. Part I : The uses of theory », Studies in History and Philosophy of Science, vol. XXIII, 4, p. 625-656.

WARwICK (A.), 1993, « Cambridge mathematics and Cavendish physics. Cunningham, Campbell and Einstein's relativity theory 1905-1911. Part II : Comparing traditions in Cambridge physics », Studies in History and Philosophy of Science, vol. XXIV, 1, p. 1-25. 